of clones with increased virulence, resistance to environmental factors and antibiotics. One of the new molecular methods is INDEL-typing which is based on the search for spontaneous inserts/deletions of several nucleotides that differ in length in different clones.

Vibrio parahaemolyticus is a common and important pathogen that causes human gastroenteritis worldwide. We have developed a method for typing $V$. parahaemolyticus strains based on the analysis of six INDEL-locus (S.O. Vodop'yanov et al., 2016). The INDEL analyses of the $V$. parahaemolyticus collection revealed that strains of different INDEL-genotypes circulate in environment. The discriminating power of INDEL-typing for environmental strains was 0.95 . However, to date, there is no information about the INDEL-genotypes of clinical strains.

The aim of this work was to study INDEL-markers of $V$. parahaemolyticus strains isolated during two foodborne disease outbreaks in the Russian Federation.

It was investigated 29 clinical strains of $V$. parahaemolyticus isolated in July-October 2012 in the Primorsky region of the Russian Federation. The study was performed on INDEL loci Vp967, Vp08, Vp619, Vp2256, VpA472, Vp506. The result showed that all 29 studied V. parahaemolyticus strains had identical INDEL genotype with the formula Vp967 - 112, Vp08 89, Vp619 - 114, Vp2256 - 111, VpA472 - 95 and Vp506 79 base pairs. Thus, both outbreaks were caused by one clone of the pathogen. At the same time, strains with other INDEL genotypes circulated in the environment.

In our opinion, the INDEL-typing method of $V$. parahaemolyticus strains can be useful in carrying out epidemiological investigation of outbreaks of food gastroenteritis.

\section{EXPRESSION OF RECOMBINANT NS1 PROTEINS OF WEST NILE, DENGUE AND ZIKA FEVER VIRUSES IN NICOTIANA TABACUM FOR FUTURE USE} IN DIAGNOSTICS

A.S. Dolgova', I.A. Goptar ${ }^{1,2}$, V.P. Bulanenko ${ }^{1}$, A.S. Pushin ${ }^{3}$, T.Y. Mitiouchkina ${ }^{3}$

${ }^{1}$ Central Research Institute of Epidemiology, Moscow, Russia; ${ }^{2}$ Research Institute of Occupational Health, Moscow, Russia; ${ }^{3}$ Branch of Shemyakin Institute of Bioorganic Chemistry of the RAS, Pushchino, Russia

In connection with the increasing frequency of infectious diseases outbreaks caused by arboviruses, the monitoring of the epidemiological situation in the Russian Federation requires development of immunological diagnostic kits for differential diagnosis. These kits could be developed using individual recombinant antigen proteins of selected viruses. Standard eukaryotic systems, for example insect cells, have a number of limitations in terms of productivity and costs. In our work, we used plants for the production of flavivirus antigens which are an ideal biofabric system because of their ability to generate large amounts of proteins with low cost and to produce an appropriate post-translational modification of recombinant proteins. Protein targets for expression were NS1 non-structural proteins of flaviviruses which were described in the literature as reliable serological markers.

The sequences of the NS1 proteins of Zika virus (ZIKV), West Nile virus (WNV) virus and the two serotypes of Dengue virus (DNV1 and DNV3), have been optimized for expression of the target proteins in the Nicotiana tabacum. The resulting DNA sequences were submitted in the GenBank database under accession numbers: MH134590, MH134591, MH134592, MH134593 for ZIKV, DNV1, DV3 and WNV respectively. Sequences were synthesized de novo using oligonucleotides by the enzymatic "Two step PCR" method.
Expression cassettes containing 35S CaMV promoter and tNOS terminator for strong constitutive expression of the target were constructed on the base of the pBI121 plasmid. Four binary vector systems for the expression of NS1 proteins in plants were developed. Tobacco leaf discs were transformed using Agrobacterium tumefaciens Ti-plasmids of strain AGL0 and further regeneration of tobacco plants was carried out. For each expression structure, 10 independent transgenic lines were obtained and were transferred to rooting media for further transfer to conditions of closed soil, which would enable the collection of the necessary amount of biomass to isolate antigen proteins, for their further use in the creation of diagnostic systems. The target gene insertions in each line were confirmed by PCR.

Thus, the plant expression system of West Nile, Dengue and Zika virus antigens was developed and our future studies would include purification of target antigens and their verification as serological markers in diagnostic systems (ELISA, immunochip).

This study was supported by the RSF grant \#17-75-10093.

2.5 doi: 10.15789/2220-7619-2018-4-2.5

\section{WHOLE-GENOME SEQUENCING AS A TOOL} FOR COMPREHENSIVE ASSESSMENT \section{OF THE PATHOGENIC POTENTIAL OF ANCIENT} ARCTIC MICROBIOMES

A.E. Goncharovi,2,3, V.A. Krylenkov ${ }^{3}$, V.V. Kolodzhieva', V.Yu. Khoroshilov ${ }^{1}$, L.A. Kraeva ${ }^{4}$, G.A. Gorbunov ${ }^{5}$

${ }^{1}$ North-Western State Medical University named after I.I. Mechnikov, St. Petersburg, Russia; ${ }^{2}$ Institute of Experimental Medicine,

St. Petersburg, Russia; ${ }^{3}$ St. Petersburg State University, St. Petersburg, Russia; ${ }^{4}$ St. Petersburg Pasteur Institute, St. Petersburg, Russia; ${ }^{5}$ Arctic and Antarctic Research Institute, St. Petersburg, Russia

Arctic permafrost is a natural reservoir of ancient prokaryotic mobile genetic elements (MGE) associated with pathogenicity or resistance to antimicrobials. It has been shown that ancient MGE have possibility to integration and effective expression in the genomes of modern bacteria. For example, an ancient Psychrobacter sp. pKLH80 plasmid from strain isolated in the Pleistocenic permafrost, contains blaRTG-6 $\beta$-lactamase gene, able to be mobilized in the modern epidemic Acinetobacter baumannii (Petrova M. et al., 2014). Horizontal genetical transfer of virulence and antibiotic resistance determinants from ancient microorganisms can lead to the appearance of genotypes with high epidemic potential. Thus the process of removal of paleomicroorganisms or their genetic material by degradation of permafrost due the global climatic changes is associated with the risk of emergence of new pathogenes or activation of forgotten infectious diseases. An effective monitoring of the pathogenic potential of the polar microbiota should be implemented.

In our opinion, one of the most promising approaches to the study of the pathogenic characteristics of bacteria found in permafrost is the whole genome sequencing. As a result of our team's studies several bacterial genomes isolated from Pleistocenic mammoth fauna were annotated. In particular, the ancient genomes of Enterococcus sp. (GenBank Acc. No. LGAN000000000000, NZ_LGAO00000000), Arthrobacter sp. ((Acc. No. QDAE00000000), Clostridium perfringens (Bac. No. QDAE00000000, QDAF00000000), Serratia spp. (Acc. No. MQRH00000000, MQML00000000), Acinetobacter lwoffii (Acc. No. LZDF00000000) were described.

The presence of of the modern epidemic clones markers in the genomes of Arctic paleobacteria was found. For example, IS16 genetic element characteristic for modern vancomycin-resistant enterococci in the ancient E. faecium 
58m strain (GenBank Acc. No. LGAN00000000) genome was detected. Also blaOXA-335 beta-lactamase gene was identified in the genome of the strain $A$. lwoffii $51 \mathrm{~m}$ (Acc. No. LZDF00000000) from mammoth intestinal tract (Goncharov A. et al., 2016). Based on this data we believe that the formation of epidemic clones in opportunistic bacteria is more likely determined by the natural selection of strains that carry genetic determinants of pathogenicity or resistance to antimicrobial drugs from natural populations (including in the polar regions of our planet), than by their formation in human society de novo.

2.6 doi: 10.15789/2220-7619-2018-4-2.6

\section{THE RESULTS OF THE RESEARCH OF USING} A COMMERCIAL KIT FOR DETENTION THE RABIES VIRUS'S RNA IN THE COURSE OF EVALUATION OF THE INFECTION OF THE FIELD MATERIAL

E.A. Gradoboeva ${ }^{1}$, E.M. Poleshchuk ${ }^{2}$, A.A. Pogoda ${ }^{3}$, G.N. Sidorov ${ }^{2}$, I.V. Deriglazov ${ }^{4}$

${ }^{1}$ Omsk State Medical University, Omsk, Russia; ${ }^{2}$ Omsk Research Institute of Natural Focal Infections, Omsk, Russia, ${ }^{3}$ Ltd "Fractal Bio", St. Petersburg, Russia; ${ }^{4}$ Centre of Hygiene and epidemiology in Omsk Region, Omsk, Russia

There are 3000 animal diseases of the Rabies registered annually in Russia (Poleshchuk et al., 2013). The monitoring of this disease helps to reduce it for animals and people. Forward-looking molecular genetic methods are used more and more often in order to diagnose the disease (Iovleva et al., 2012; Devyatkin et al., 2014; Dedkov et al., 2016).

"The kit for the detection of the Rabies virus's RNA in a complete set" produced by Ltd "Fractal Bio" (St. Petersburg) is a set of reagents for the outflow of the nucleic acid and the realization of the real-time PCR with hybridization-fluorescent registration, it was used to analyze the Rabies of 210 samples: 153 field material and 57 samples of bioprobes.

The initial material included samples of foxes, racoon dogs, corsac foxes, minks, Siberian striped weasels, martens, ferrets, sables, ermines, bats and men as a $10 \%$ suspension of a cerebrum based on Henx solution. There were also the samples of bioprobes of not purebred white mice that died in the course of the experiment. The samples were collected from 2-11 passages and were presented by $10 \%$ suspensions of the cerebrum. The apparatus used during the research was RotorGene6000.

With the use of test-system, the presence of Rabies virus's RNA was confirmed in all positive samples of the initial material of foxes $(n=14)$, cattle $(n=6)$ and men $(n=$ 2). None was detected in all the negative samples. If negative probes, they registered the formation in the reaction of the product of the amplification of the internal control sample (genomic DNA of mammals).

A different selection of the field material revealed drawbacks of the set when using the internal control sample (ICS). Therefore, ICS did not work with the initial samples of racoon dogs $(n=10)$, bats $(n=12)$ and all the mice samples. When researching the initial material of foxes $(n=35)$ and corsac foxes $(n=3)$, there was no reaction of the ICS or it was very weak (from the $30^{\text {th }}-36^{\text {th }}$ cycle) in more than $50 \%$ cases. The dilute of the nucleic acid, repeated several times, did not improve the result of the reaction with the ICS. That means it lacks the universality of the amplifiable fragment. Provided that there is high reliability of identification of the Rabies virus's RNA, the negative results of the ICS make the interpretation of the result more difficult.

Therefore, the above-mentioned test-system needs refining in part of the optimization of the ICS. doi: 10.15789/2220-7619-2018-4-2.7

\section{COLLISION OF CRISPR-CAS SYSTEMS WITH THE POTENTIAL OF VIRULENCE OF ESCHERICHIA COLI STRAINS THAT PRODUCE SHIGA-TOXINS}

E.I. Ivanova ${ }^{1}$, N.A. Arefieva ${ }^{2}$, Yu.P. Dzhioev ${ }^{3}$, T.V. Tunik ${ }^{1}$, A.Yu. Borisenko ${ }^{3}$, L.A. Stepanenko ${ }^{3}$, E.A. Kungurtseva ${ }^{1}$, E.V. Grigorova ${ }^{1}$, U.M. Nemchenko ${ }^{1}$, L.V. Rychkova ${ }^{1}$ ${ }^{1}$ Scientific Center for Family Health and Human Reproduction Problems, Irkutsk, Russia; ${ }^{2}$ Irkutsk State University, Irkutsk, Russia; ${ }^{3}$ Research Institute for Biomedical Technologies of Irkutsk State Medical University, Irkutsk, Russia

Shiga toxin-producing Escherichia coli (STEC) strains are a diverse group of $E$. coli strains belonging to over $400 \mathrm{E}$. coli serotypes, some of which cause outbreaks and sporadic cases of food-borne illnesses ranging from diarrhea to hemorrhagic colitis and the hemolytic-uremic syndrome (HUS). It was long believed that bacteria could not resist phage attacks, but in 1987 a strange region was discovered in the $E$. coli genome that consisted of multiple repeats. The discovered structures were termed CRISPR-Cas (Clustered Regularly Interspaced Short Palindromic Repeats - CRISPR-associated proteins). CRISPR is a chronological record of infectious assault on a bacterium from viral and other genetic elements. According to Delannoy S. et al., It has been shown that CRISPR polymorphisms in $E$. coli strongly correlate with both the serotype of the microorganism and the presence of virulence factors in its genome (stx and eae genes).

Genomic sequences (GenBank databases) of $E$. coli isolates of different serogroups $(n=658)$ were analyzed for the presence of CRISPR-Cas systems and stx-genes. Of the 658 $E$. coli isolates, $60.5 \%$ of the loci of CRISPR-Cas systems were found. At the same time, according to the structural organization of CRISPR-Cas, the systems of the strains studied were of type I-E in $92.7 \%$ and type I-F of $7.3 \%$. Analysis for the presence of genes of shiga toxins 1 and 2 types showed that $14.4 \%$ of isolates having a CRISPR-Cas system of type I-E were positive. The genes $s t x 1 A$ and $s t x 1 B$ were registered in 6.1 and $5.9 \%$ of cases. The frequency of registration of stx2 subtypes was 2 times higher than stx 1 (6.1 and 12.3\%). Stx $2 B$ was detected in $9.9 \%$ of cases.

Stx $2 A$, which according to the literature is more often associated with HUS than other subtypes, was detected in $9.7 \%$. According to the CRISPR-Cas data, the I-E subtype system is associated with the $\operatorname{stx} 1 A(10.1 \%)$, stx $1 B(9.8 \%)$ stx $2 A(16.1 \%)$ and stx $2 B(16.3 \%)$ genes. Further linkage between CRISPR elements and the pathogenicity of the isolate will allow us to determine the causal relationships that stimulate the acquisition of the isolate of both the CRISPR-Cas system and the genes coding for pathogenicity.

\section{NGS CAPABILITIES FOR THE STUDY OF ENTEROAGGREGATIVE E. COLI}

A.A. Kechin ${ }^{1,2}$, D.S. Boldyreva ${ }^{1,2}$, M.A. Makarova ${ }^{3}$, L.A. Kaftyreva ${ }^{3}$, S.G. Scherbak ${ }^{4,5}$, S.V. Apalko ${ }^{5}$, M.L. Filipenko ${ }^{1,2}$, A.M. Sarana ${ }^{4,5}$, M.A. Churina ${ }^{5,6}$ ${ }^{1}$ Institute of Chemical Biology and Fundamental Medicine of the Siberian Branch of the Russian Academy of Sciences, Novosibirsk, Russia; ${ }^{2}$ Novosibirsk State University, Novosibirsk, Russia, ${ }^{3}$ St. Petersburg Pasteur Institute, St. Petersburg, Russia; ${ }^{4}$ St. Petersburg State University, St. Petersburg, Russia; ${ }^{5}$ City Hospital No. 40 of the Resort Administrative District, St. Petersburg, Russia, ${ }^{6}$ St. Petersburg Clinical Infectious Diseases Hospital named after S.P. Botkin, St. Petersburg, Russia

Enteroaggregative $E$. coli (EAggEC) are the causative agents of such intestinal diseases as acute and chronic diarrhea, inflammation of the intestine in children and 